\title{
A Study on Comprehensive Evaluation of C2C E-commerce Website Competitiveness
}

\author{
Ming Li \\ JiLin Business And Technology College, ChangChun, China \\ 120857715@qq.com
}

Keywords: C2C, E-commerce Website, Competitiveness, Evaluation Research.

\begin{abstract}
C} 2 \mathrm{C}$ e-commerce websites have developed rapidly with intensive competition. For C2C E-commerce websites, the prerequisite for promoting enterprise's competitiveness level and achieving competitive advantages is to assess and acquaint their competitiveness, which is also a significant problem to be solved at present. Based on the analysis of E-commerce website evaluation, the competitive model of C2C E-commerce website is established, and the evaluation index system of C2C E-commerce competitiveness is also built and then the comprehensive evaluation method based on AHP method and multi-level fuzzy comprehensive evaluation are proposed. The results show that the impact of experts' subject opinions during the evaluation can be effectively reduced by evaluation index system and comprehensive evaluation method which is an effective and feasible method to evaluate the competitiveness of C2C E-commerce websites.
\end{abstract}

\section{Introduction:}

C2C E-commerce pattern refers to an online trading model where network service providers offer the paid or unpaid using of E-commerce platforms and trading programs through computer and network technology to allow the trading parties (mainly for individual users) to bid and bargain independently on their own platforms.

Nowadays, there is fierce competition in the area of C2C E-commerce, and thus how to grasp the great business opportunities E-commerce development has presented and how to improve competitiveness and obtain competitive advantages during the $\mathrm{C} 2 \mathrm{C}$ E-commerce are the urgent issues that should be solved. In order to ultimately improve the competitiveness, each C2C E-commerce website should firstly face the comprehensive evaluation of the competitiveness, that is to answer the basic questions of whether their websites have competitiveness or not, where their competitiveness is and how strong their competitiveness is. The only way for C2C E-commerce websites to know the key specialties and disadvantage is to evaluate the competitiveness comprehensively so that they can purposefully adjust their business processes and allocation of resources and guide the strategic direction during the business development process. E-commerce website evaluation is one of the research hot spots in the field of electronic commerce. The existing work is concentrated on the evaluation of B2B[1], B2C and B2G E-commerce websites and researchers have proposed a variety of evaluation methods which have certain reference significance for studying the evaluation of C2C E-commerce competitiveness. Comprehensive evaluation method refers to that the weighted comprehensive evaluation system should be established firstly and the date should be collected through the technical measurements, expert surveys, user surveys and other methods and then the comparative analysis and model analysis should be used to mine and analyze the data and relative information. This paper mainly studies the comprehensive evaluation of C2C E-commerce website competitiveness, aiming to establish a comprehensive evaluation index system of C2C E-commerce website competitiveness and propose the feasible evaluation methods. 


\section{The Evaluation Index System of C2C E-commerce Website Competitiveness}

The evaluation index system is the basis and key point of comprehensive evaluation system. Through the analysis on the evaluation methods of current E-commerce websites, the first level target of $\mathrm{C} 2 \mathrm{C}$ E-commerce website competitiveness is determined as the security, service, information, technology and brand of the website after combining with the characteristics of Taobao, Amazon and other C2C E-commerce web sites and then the second level target is obtained through decomposition and refinement so that a evaluation index system that is suitable for the C2C E-commerce website competitiveness is constructed (As shown in Figure 1).

Security (A1). The security of C2C E-commerce website mainly includes privacy (B1), network security (B2) and network accreditation (B3)[2].

Service (A2). The services of C2C website are: Communication (B4) and transaction (B5). Thereinto, the transaction includes the secure payment service, logistics system (B6), return or exchange service (B7) and credit evaluation system (B8).

Information (A3). Evaluation criterion and specific indexes include the following five aspects: practicality (B9), comprehensiveness (B10), accuracy (B11), novelty (B12).

Technology (A4). From the perspective of consumer evaluation, the technical indexes of C2C E-commerce website include: Website response speed (B13), user interface (B14), retrieval pathway (B15) and retrieval results (B16).

Brand (A5). The influencing factors of brands include: Website visibility (B17), network influence (B18), market share (B19).

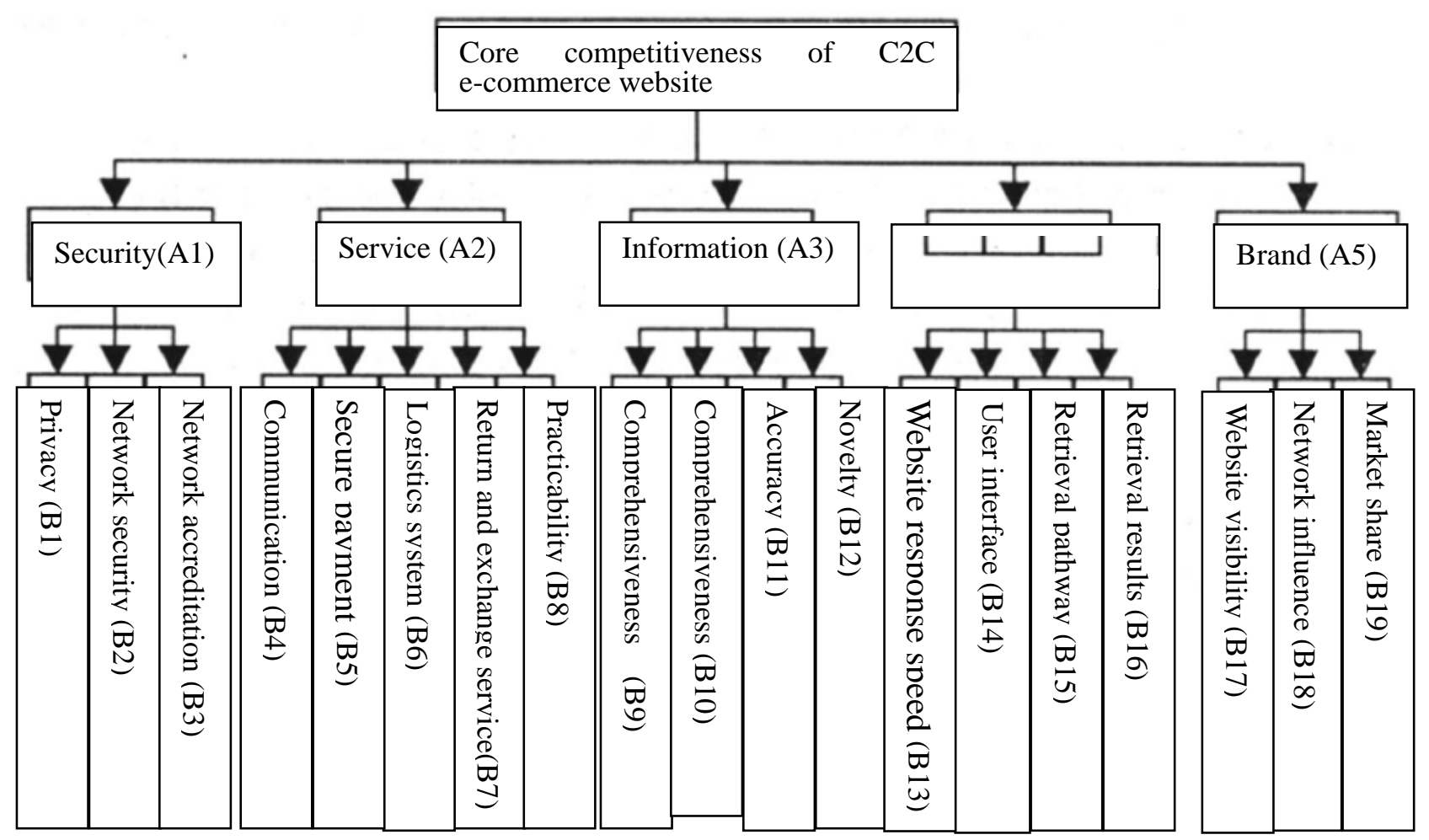

Figure 1: Evaluation Index System of C2C E-commerce Website Competitiveness

\section{The Comprehensive Evaluation Method of C2C E-commerce Website Competitiveness}

The AHP method is used to calculate the index weight which means that the scoring criteria is used to judge and establish the judgment matrix between the indexes after the pairwise comparison of the elements of secondary index(Comparative between the degree of importance and the that of unimportance) with a certain element of the first index as the evaluation criteria and the judgment 
matrix should be calculated and finally the index weight is obtained. The scoring criteria of AHP method was proposed by Satty, the founder of AHP which used the equality, slight importance, importance, much importance, extreme importance to distinguish relative importance between the two indexes. When the higher precision is needed, the comparison can be made between the adjacent standards and thus there are nine criteria in total (extreme importance, much importance, importance, slight importance, equality, slightly less importance, no importance, no much importance, not very importance). These criteria not only maintain the consistency, but also can be applied in practice easily. The first evaluation index in the evaluation index system of C2C E-commerce website in Figure 1 is taken fox example. Through user survey and data analysis, it is found that safety (A1) is slightly more important than service (A2), scored 2; and safety is more important than information (A3), scored 4; slightly more important than technology (A4), scored 3; more important than brand (A5), scored 5. Then the judgment matrix of first level can be established, as shown in Table 1.

Table 1 Judgment Matrix of C2C E-commerce Website Competitiveness

\begin{tabular}{l|llllll}
\hline & A1 & A2 & A3 & A4 & A5 \\
\hline A1 & 1 & 2 & 4 & 3 & 5 \\
A2 & $1 / 2$ & 1 & 3 & 2 & 4 \\
A3 & $1 / 4$ & $1 / 3$ & 1 & $1 / 2$ & 2 \\
A4 & $1 / 3$ & $1 / 2$ & 2 & 1 & 3 \\
A5 & $1 / 5$ & $1 / 4$ & $1 / 2$ & $1 / 3$ & 1 \\
\hline
\end{tabular}

The weight of each index can be obtained through using AHP method: $W 1=0.42$. W2 $=0.26$, $\mathrm{W} 3=0.1, \mathrm{~W} 4=0.16$, W5 $=0.06$. The maximum characteristic roots: $\lambda \max =5.0674$.

After the consistency checking of the above results, it is concluded:

$\mathrm{CI}=\mu=\frac{\lambda \max -n}{n-1}=\frac{5.0674-5}{5-1}=0.01685$.

Looking up the table, it is obtained RI $=01122$.so

$\mathrm{CR}=\frac{C I}{R I}=\frac{0.01685}{1.12}=0.015<0.10$.

Therefore, $\mathrm{X}$ has satisfactory consistency.The relative weight of each level can be finally obtained by using AHP method through establishing judgment matrix of each level index. Finally, the weight of competitiveness evaluation index system of the whole C2C E-commerce website can be obtained, as showed in figure 2; thereinto, the calculating of the relative weight of the secondary index is similar to that of the first one, which is not explained here[3].

Table 2 Weight of Competitive Evaluation Index of C2C E-commerce Website

\begin{tabular}{l|l|l}
\hline First Index (Ai) & Secondary Index(Aij) & $\begin{array}{l}\text { Synthetic } \\
\text { Weight(W) }\end{array}$ \\
\hline A1 Safety(0.42) & B1 Privacy (0.35) & 0.147 \\
& B2 Network security(0.35) & 0.147 \\
& B3 Network authentication(0.3) & 0.126 \\
\hline A2 Service(0.26) & B4 Communication(0.15) & 0.039 \\
& B5 Payment platform(0.25) & 0.065 \\
& B6. Logistics system (0.2) & 0.052 \\
& B7 Returning or replacing goods & 0.039 \\
& service(0.15) appraisal system & 0.065 \\
& B8 Credit and apr \\
& $(0.25)$ & \\
\hline
\end{tabular}




\begin{tabular}{l|l|l}
\hline A3 Information(0.1) & B9 Practicability(0.3) & 0.03 \\
& B10 Comprehensiveness(0.2) & 0.02 \\
& B11 Accuracy(0.25) & 0.025 \\
& B12 Novelty(0.25) & 0.025 \\
\hline A4 Technology(0.16) & B13 Website response speed(0.25) & 0.04 \\
& B14 User interface(0.3) & 0.048 \\
& B15 Retrieval methods(0.15) & 0.024 \\
& B16 Retrieval results(0.3) & 0.048 \\
\hline A5 Brand(0.06) & B17 Network visibility(0.2) & 0.012 \\
& B18 Web impact(0.3) & 0.018 \\
& B19 Market share(0.5) & 0.03 \\
\hline
\end{tabular}

Using the multi-level fuzzy comprehensive evaluation method to evaluate the competitiveness of E-commerce website. Multi-level fuzzy comprehensive evaluation method is a bottom-up evaluation method which is calculated layer by layer. The basic idea is: to start from the bottom index and determine the membership of bottom index after knowing the relative weight of each index, and then to use the transformation principle of fuzzy set to construct the fuzzy evaluation matrix, using the membership to describe fuzzy boundaries of the factors, and finally obtain the evaluation result of the object through multilayer composite operation. Multi-level fuzzy comprehensive evaluation method does not give the evaluation level directly, instead it often requires the users to define in terms of field characteristics.

\section{References}

[1] H. Zhu. An Empirical Research on Technology Spillovers of Subsidiaries of Multinational Companies in China. Science Research Management, 2003(3): 76.

[2] H.Y. Zhang. Foreign Technology Diffusion and Development of High-tech Industry in Hubei Province)))Taking Optical Communication Industry in Wuhan for example. Studies in Science of Science, 2006(2): 68.

[3] X.T. Zhang .Application of Quantitative Economics. Beijing: China Machine Press,2009. 